\title{
Patienten erzählen in der psychoanalytischen Beziehung - Faktualität und Fiktionalisierung
}

\author{
Brigitte Boothe
}

Nichts ist so schwierig wie Gerechtigkeit gegen die Tatsachen

(Wittgenstein 1930)

\section{$\ddot{U b e r s i c h t ~}$}

In den achtziger und neunziger Jahren des vorgegangenen Jahrhunderts erzählten junge Patientinnen ihrem Psychoanalytiker Freud in verstörender Häufigkeit von sexuellen Übergriffen, die sie als Kinder und Jugendliche durch männliche Verwandte erlitten hatten. Freud ging zunächst von der Faktizität der Schilderungen aus, doch gelangte er schließlich - das war ein wesentlicher Schritt auf dem Weg zur Psychodynamik des Unbewussten - zu einer anderen Auffassung: Die Patientinnen, so vermutete er jetzt, schilderten vielfach nicht, was sich real zugetragen hatte, sondern was einem unbewussten Darstellungsanliegen entsprach. Die Erzählungen verweisen in der neuen Sicht Freuds nicht auf äußeres Geschehen, sondern auf den Autor, nicht auf historische Faktizität, sondern auf Leistungen der Fiktionalisierung im biografischen Gestalten. Freuds Hinterfragung der historischen Wahrheit bestimmter Missbrauchserzählungen sorgt zwar bis heute für moralische Irritation in der Fachöffentlichkeit, doch öffnete jene frühe Exploration biografischer Fiktionalisierung den Blick für die imaginativen und dramaturgischen Konstruktionen des Erinnerns und Erzählens. Erzähler im Alltag und in der Psychotherapie sind tendenziell von der Faktualität ihrer biografischen Schilderungen überzeugt, auch fordern sie die Glaubensbereitschaft des Hörers. In der Tat behandelt man in der alltäglichen und der psychotherapeutischen Interaktion biografische Erzählungen nicht in einer Haltung kritischen Hinterfragens oder reflexiver Distanz. Vielmehr gehören glaubensbereite Affirmation und artikulationsbereite emotionale Teilnahme zu dem, was man als narrativen Takt oder narrative Feinfühligkeit des Hörers bezeichnen könnte. Hörer erwarten, dass ihr gläubiges Vertrauen in die Wahrheit der Erzählung - die sie nur in Ausnahmefällen kontrollieren - honoriert wird. Hörer richten sich intuitiv auf das Befinden und das Selbstgefühl des Erzählers aus oder auf - eine schillernde Wortprägung - seine „psychische Wahrheit“. Da Psychotherapeuten und Psychiater heute sensibel für die Verflechtung von Faktualität und Fiktionalisierung in der Struktur biografischer Darstellungen sind, verstehen sie diese nicht in erster Linie als Schlüssel zu stichfesten anamnestischen Informationen, sondern als Königsweg zum narrativen Selbstentwurf, zur mentalen Verfassung, zur Kon- 
flikt- und Abwehrdynamik. Biografische Erzähler beuten gewöhnlich die Bereitschaft der Hörer, ihnen zu glauben, nicht aus; dennoch verwandeln sie Geschichte in einen persönlichen Kosmos, ein eigenes Reich im Dienst des Selbstgefühls und des psychischen Komforts. Anders formuliert wird das faktisch Gegebene im Modus der Nachträglichkeit - den eigenen Präferenzen und Relevanzen einverleibt und als narrativer Kosmos zur Anschauung gebracht.

Die Erzählung genügt vier Funktionen: zum einen der Vergegenwärtigung (Vergangenes wird aktualisiert und dabei Bedingungen der Gegenwart adaptiert), zum zweiten der sozialen Integration (der Erzähler wirbt um Anerkennung), zum dritten der Bewältigung (die nachträgliche Regieführung schafft Kontrolle), zum vierten der Wunscherfüllung (die Erzählung folgt, einem guten oder schlechten Ende zusteuernd, einem Wunsch- oder hedonischen Imperativ). Erzählungen können in den einzelnen Funktionen scheitern, beispielsweise kann die Erzählung Befremden wecken statt Empathie, Irritation statt Zustimmung.

Was die Aktualisierungsleistung der Erzählung angeht, so baut hier der Hörer auf etwas wie Verantwortungsübernahme seitens des Erzählers. Wird etwa eine Figur als maligner Offender in der Darstellung eines sexuellen Übergriffs eingeführt, also einer Handlung bezichtigt, die ernste lebenspraktische Folgen hat, so erwartet man - und überprüft gegebenenfalls auch - narrative Redlichkeit und die Fähigkeit des Erzählers, eine zuverlässige faktuale Darstellung zu geben: Ein auch heute noch außerordentlich stimmiges und psychologisch überzeugendes Porträt eines kindlichen Erzählers, dem man zu Unrecht glaubt, findet sich in Gottfried Kellers Kapitel „Kinderverbrechen“ aus seinem Roman Der grüne Heinrich (1789).

Ein ausführliches Psychotherapie-Beispiel für irritierende Fiktionalisierung im Erzählgeschehen wird dargestellt und im Blick auf psychotherapeutische Praxis diskutiert. Narrative, die im Kontext prekärer Fiktionalisierung irritieren oder befremden, sind entscheidende Risikofaktoren für das Zustandekommen einer therapeutischen Bündnisbildung; sie konfrontieren das therapeutische Gegenüber mit hohen Ansprüchen an seine Fähigkeiten, den Ratsuchenden kommunikativ zu erreichen. Wie psychotherapeutische Kooperation im Zusammenspiel von narrativer Empathie und Herausforderung zur Reflexivität gelingen kann, wird abschließend diskutiert.

\section{Die Entdeckung narrativer Fiktionalisierung in der Frühzeit der Psychoanalyse}

Freud (1897) berichtet im Brief vom 21.9.1897 an seinen Freund Wilhelm Fließ davon, dass er seine Neurotica nicht mehr für zuverlässige Erzählerinnen halte. Oft schilderten sie sexuellen Missbrauch, den sie vorgeblich in früher Jugend durch männliche Verwandte erlitten hatten, doch habe er den Eindruck, dass sie unbewusst vielfach sich selbst als naive Opfer, die Erwachsenen hingegen als Täter inszenierten, und dies als Ausdruck latenter Konfliktdynamik: Sie selbst hatten, so 
Freuds Vermutung, im frühen Kindesalter ein väterliches Liebesobjekt begehrt und auf kindliche Weise erotisches Verlangen inszeniert; dieses Begehren und diese Initiative aber waren verboten, in der Vorstellungswelt des Kindes handelte es sich um die Verletzung eines Tabus, auch wenn der Erwachsene auf die kindlichen Avancen nicht missbräuchlich reagierte. Für das Kind war nicht nur ein Tabu im Spiel, sondern auch eine kränkende Zurückweisung. In der sich weiter entwickelnden mentalen Welt der Heranwachsenden war, in der neuen Sicht Freuds, der infantile Konflikt um Schuld und Kränkung, ins Unbewusste verdrängt, noch wirksam. Verdrängte Bilder von erregender körperlicher Vereinigung und erfüllender Intimität, aber auch von beschämender Zurückweisung und Verurteilung, blieben im psychischen Haushalt als wunscherfüllendes Regulativ wirksam. Die Jugendliche oder die Erwachsene - realitätsorientiert; ihr Denken ist in der Diktion Freuds dem Sekundärprozess verpflichtet - verfügen über Praxiswissen, Reflexivität und emotionale Intelligenz. Die imaginative - dem in der Diktion Freuds (1900; zur Definition des Primärvorgangs: Kap. VII. „Zur Psychologie der Traumvorgänge“, S. 489; zu einigen Charakteristika von Primärvorgängen: Kap. VI. „Die Traumarbeit“, S. 234-236; auch S. 265-266) dem Primärprozess verpflichtete - Verstandestätigkeit des Kindes scheint längst überwunden, abgetan und versunken. Doch macht sie sich unbewusst, auf dem Weg der handelnden und der sprachlichen Inszenierung, in verkleideter Form deutlich (Leichsenring 2014). Wenn eine junge Patientin von sexuellen Übergriffen erzählt, die sie in früher Jugend habe hinnehmen müssen, kann die Erzählerin von der Wahrheit der Geschichte überzeugt sein, auch wenn sie sich als fiktive Erzählung herausstellt. Wenn sie zur Sprache bringt, wie sie ahnungslos das Verlangen Erwachsener weckte und schuldlos im Verborgenen und Geheimen zu sexuellen Handlungen genötigt wurde, ist auf der Ebene konfliktdynamischer Verhältnisse auf mehrfache Weise Entlastung gelungen:

- Sie erfährt Schuldentlastung: Schuld haben andere.

- Kränkung ist repariert: Sie hat sexuelles Begehren geweckt, statt Zurückweisung zu erfahren.

- Exklusive Intimität wird geboten: Ein erregendes Geheimnis ist zu wahren.

- Auch ein Bedürfnis nach Strafe wird reguliert: Sie hat Entblößung und Fremdbemächtigung hinnehmen müssen.

Ausdrücklich ist hervorzuheben, dass die nachträgliche narrative Umarbeitung nur dann entlastungsfunktional ist, wenn sowohl die Erzählerin wie auch der Hörer die Darstellung für wahr halten.

\section{Unter der Herrschaft der Wünsche}

Eine erste Standortbestimmung:

- Biografische Erzähler stehen häufig unter der Herrschaft unbewusster Wunschund Angstvorstellungen, die sich in der nachträglichen narrativen Gestaltung 
erfahrener Lebenswirklichkeit verdeckt geltend machen, so dass die erzählende Person ihrer nicht gewahr wird. Sie erzählt, was sie für wahr hält, aber auch, was sie glauben will.

- Erzähler schildern Erlebtes unter dem Einfluss von Darstellungsanliegen, die sich zwar in der Erzählung als Fiktionalisierungsleistungen geltend machen, aber als solche nicht erkannt werden.

- Biografische Erzähler haben ein „Wirklichkeitsgefühl“ (Botella 2005), und sie wollen - so auch Freuds junge Patientinnen - Zustimmung für ihre Erzählungen.

- Das „Wirklichkeitsgefühl“ spielt dabei eine entscheidende Rolle: Wenn die Patientinnen schuldlose Opfer waren, dann sind sie vom inneren Konflikt zwischen Begehren und Strafangst vorübergehend entlastet, auch auf sozialer Ebene, wenn die Erzählung Glauben findet.

- Freud versagte offenbar bestimmten Erzählerinnen Zustimmung, dies bedeutet das Risiko, mindestens einigen Erzählerinnen Unrecht getan zu haben. Unrecht geschieht bekanntlich auch, wenn eine Person fälschlich des missbräuchlichen Handelns bezichtigt wird.

- Dabei muss es - das sollte deutlich geworden sein - nicht um eine direkte oder bewusste Lüge gehen; vielmehr legen Freuds voraussetzungsvolle, seinerzeit neue und anstößige Ideen nahe, dass eine unbewusste Konflikt- und Abwehrdynamik zur fiktionalisierenden Modellierung des biografischen Narrativs führt.

- Die Idee der fiktionalisierenden Modellierung von Erzählungen, die beanspruchen, einen realen Vorfall zu schildern, gehört zum tradierten kulturellen Wissen, auch die Verwendung bestimmter narrativer Muster wie beispielsweise die Darstellung einer Täter (maligner männlicher Offender)-Opfer (benigne weibliche Geschädigte)-Konstellation. Sprichwörtlich ist die narrative Intrige von Potiphars Frau gegen Joseph, der ihre erotischen Avancen zurückgewiesen hat. Diese Erzählfigur eines fingierten Übergriffs als Rache einer Verschmähten ist in der Weltliteratur und den populären Medien variantenreich vertreten. Variantenreich ist dabei auch die den Erzählerinnen zugeschriebene Haltung. Kalkuliertes Lügen steht neben diversen Strategien mehr oder weniger bewussten oder auch nicht-bewussten Entstellens und Verzerrens.

- Biografische Erzählungen verlangen Glaubensbereitschaft und zustimmendes Wohlwollen beim Publikum. Die narrative Fehlbezichtigung kann für den vermeintlichen Offender negative lebenspraktische Folgen haben.

- Die Rache der verschmähten Frau? Das Mädchen, das den Vater anklagt? - Es ist den Erzählungen nicht anzusehen, ob oder wie weit sie den Faktenbezug verfehlen. Personen, die in Erzählungen als maligne Figuren auftraten, können zu Unrecht unter Verdacht geraten; Rezipienten von Erzählungen können zu Recht oder zu Unrecht beschuldigt werden, sich dem Ernstnehmen realer Not $\mathrm{zu}$ verweigern und vielmehr bestimmten Männerfantasien aufzusitzen, etwa seit die Psychoanalyse die Trieb- und Vorstellungswelt des Sexuellen konzeptualisiert - der Fantasie von der kastrierenden Frau. 
- Die Idee der unbewussten fiktionalisierenden Modellierung als faktual intendierter Missbrauchserzählungen geht als systematisches und theoriegeleitetes Projekt auf die Psychoanalyse zurück. Ein unbewusstes Darstellungsanliegen so und so möge es gewesen sein - wird narrativ organisiert und geglaubt, auch von der erzählenden Person selbst. Der affirmativ-persuasiv-suggestive Charakter des Erzählens bietet dem Autor oder der Autorin Ersparnis von Unlust, Unbehagen und spannungsvoller Selbstkonfrontation; biografisches Erzählen nährt Wunschdenken und steht nicht im Dienst der Selbstvergewisserung und Realitätsorientierung.

Das falsche narrative Zeugnis ist bekanntlich eine Problematik von expansiver Praxisrelevanz. Unter welchen Bedingungen, in welchen Erzählkontexten und wie weitgehend biografische Narrative von welcher Art des Faktenbezugs abweichen, ist Gegenstand öffentlicher Aufmerksamkeit, wenn man an die Thematik der - bewussten oder nicht bewussten - Falschbezichtigung, gefälschter Biografien oder induzierter Falscherinnerung denkt. Auch liegt inzwischen langjährige empirische Forschung vor, besonders reichhaltige Befunde etwa in den von Loftus (Laney und Loftus 2005) initiierten, inzwischen breit differenzierten Studien zur falschen Erinnerung.

Erzähler rechnen mit Glaubenskredit und mit der Bereitschaft des Zuhörers, an ihrer Sympathieregie zu partizipieren, ihre persönliche Perspektive zu teilen, sowie über Protagonisten oder Antagonisten gemäß den Relevanzen und Präferenzen der erzählenden Person zu urteilen. Diese narrative Urteilsbildung folgt dem Gesetz der „naiven Moral“, in der Formulierung von Jolles (1930). Die „naive Moral" orientiert sich am egozentrischen Imperativ. Sie entspringt einem Gefühlsurteil darüber, wie es in der Welt zugehen sollte, wenn es machtvolle Parteinahme für das eigene Anliegen gäbe (Boothe 2002, 2011). Engagiert für die Belange des zentralen Sympathieträgers bauen Leser Spannung auf. Die narrative Regieführung transformiert das Ereignis in eine konfigurierte Situation. Bei der narrativen Vermittlung des Gegebenen als biografische Schilderung geht es um retrospektiv zur Anschauung gebrachte Lebenswirklichkeit in der Perspektive einer Beteiligten. In der biografischen Darbietung wird diese zur Schlüssel- oder Hauptfigur. Sie tritt auf als Protagonistin in einer dramatischen Konfiguration mit Akteuren, Requisiten, Kulissen und Handlungsrepertoire. Diese Narrativierung der Vergangenheit lässt sich als elementare Verwandlung in einen individuellen Kosmos, eine Welt, organisiert im Zeichen der Relevanzen und Präferenzen der Autorin, begreifen. Das retrospektive Porträt persönlicher Lebenswirklichkeit gilt der Sprecherin oder dem Sprecher als authentisch, entzieht sich jedoch partiell den Ansprüchen der Tatsachengerechtigkeit und folgt umso mehr unbewussten Darstellungsanliegen, die sich mit Hilfe sprachlicher Fiktionalisierung geltend machen. Bei der sprachlichen Fiktionalisierung geht es nicht nur um grundsätzlich intersubjektiv überprüfbare Realitätsdivergenzen, sondern auch, im Kontext von Kindheitserinnerungen, um den möglicherweise bedeutenden Einfluss, den 
die emotionale und kognitive Verfassung des Kindes, also frühe mentale Entwicklungsstadien, auf Erinnern, Vergegenwärtigen und Darstellen ausüben. Auch die spezifische Kommunikationssituation und Maßnahmen der kommunikativen Einflussnahme auf die emotionale Parteilichkeit des Hörers und Strategien der Sympathielenkung können Fiktionalisierung faktualer Erzählungen aus der eigenen Biografie begünstigen.

\section{Faktualität und Fiktionalisierung sind noch nicht getrennt: Die Macht des kindlichen Geistes}

Die psychoanalytischen Ideen zur Kindheit waren neu, folgenreich und bleiben bis heute in vielen Forschungsfeldern fruchtbar. Es geht darum, dass die mentale Welt des Kindes anders ist als die mentale Welt des Erwachsenen. Die geistige Welt des Kindes ist in ihrer Besonderheit, unter anderem in ihrer Nähe zu Wunsch und Imagination, in ihrer Verflochtenheit von Vorstellungswelt und Realgeschehen, zu erschließen. Diese mentale Tätigkeit im Zeichen des Primärprozesses geht im Denken, Fühlen und Handeln des Erwachsenen, anders formuliert: in einer vom Sekundärprozess bestimmten geistigen Welt, nicht unter, sie entfaltet durchaus - leicht erkennbar im Traum und anderen regressiven mentalen Verfassungen (Koukkou und Lehmann 1998) oder in hoch involvierenden emotionalen Zuständen - latente Wirksamkeit, ebenso im Erzählgeschehen, weil hier individuelle Präferenzen und Relevanzen, die desiderative oder Wunschorientierung (Boothe et al. 2012 ), zur Geltung kommen.

Die Beziehungsschicksale und Erfahrungen der frühen Kindheit galten der Psychoanalyse von Beginn an als prägend für die psychische Entwicklung (Boothe 2012) und als entscheidend für die Einschätzung von Risiken, psychisch zu erkranken. Gleichzeitig bot die Frage nach der frühen Kindheit ein gravierendes Problem für Forschung und Praxis. Die retrospektiven Kindheitserzählungen der Jugendlichen und Erwachsenen sind lediglich als überformte retrospektive Kreationen oder als „Deckerinnerungen“ (Freud 1899) zugänglich. Und doch: Der Patient soll Kontakt mit seinem kindlichen Selbst herstellen können, und er soll im Prozess der Selbstexploration Einsicht in die eigene unbewusste Konfliktdynamik gewinnen. Wissenschaftsphilosophisch hat Adolf Grünbaum (1988) Freuds langjährige, differenzierte, in Skepsis mündende Auseinandersetzung mit der Unzugänglichkeit authentischer kindlicher Lebensrealität nachgezeichnet, ebenso sein letztlich vergebliches Ringen mit der Frage, ob und wie in der Beziehung zwischen Patient und Therapeut zwischen Suggestion und Einsicht zu unterscheiden sei. Längst ist die empirische Kindheitsforschung etabliert, insbesondere die direkte Beobachtung von Säuglingen und von Eltern-Kind-Interaktionen, auch bei der Entwicklung von kindlicher Erzählkompetenz im kommunikativen Zusammenspiel von Erwachsenen und Kleinkindern (Boothe 2009). Was die Frage des retrospektiven Erzählens und der erzählenden Verarbeitung von Erfahrung in der Psy- 
chotherapie angeht, bleiben die frühen psychoanalytischen Ideen zum Konstruktionscharakter (Clandinin und Connelly 2000; Fludernik 2009) des Narrativs, das Erfahrung gestalten will, dabei aber wunschgeleiteten Darstellungsanliegen genügt, bis heute wichtig.

\section{Die szenische Struktur der Erlebniswelt und die diskursive Wirklichkeit}

Flitner und Merle (1989) explorierten in detaillierter Literaturrecherche zu den Werken Freuds im Kontext der sogenannten „Verführungstheorie“ und ihrer Relativierung seit 1897 (Brief an Wilhelm Fließ; hg. Masson 1986), dass die sogenannte „psychische Realität“ und die „szenische Struktur der Erlebniswelt“ bereits in den frühen neunziger Jahren des 19. Jahrhunderts begrifflich relevant waren (Flitner und Merle 1989: 252). Für die psychologisch und psychophysisch wichtige Ebene der Regulierung des Befindens und der kognitiven Organisation ist die narrative Strukturierung ein zentrales Moment (Sarbin 1986: 9). Schafer (1995) und Spence (1984), herausragende Repräsentanten einer narrativen Orientierung in der Psychoanalyse, legten die elaborierte Erschließung von sprachlichen Thematisierungs- und Darstellungsverfahren sowie interaktiven und kommunikativen Inszenierungen vor. Jenseits faktischer oder historischer Wahrheit rückte die persönliche oder „psychische“ Wahrheit des Erzählers ins Zentrum therapeutischer Aufmerksamkeit. „Psychische Wahrheit“ bleibt bis heute eine schwer zu fassende Wortprägung. Ihre Bedeutung mag sich unter Verweis auf das Paradigma des Konstruktivismus erhellen: „Im sozial- und kulturwissenschaftlichen Diskurs der letzten Jahre“, schreibt Deppermann (1998: 86), verliere „die für die Moderne grundlegende Unterscheidung ,authentisch' vs. ,inszeniert', ,real' vs. ,fiktional', ,aufrichtig' vs. ,unaufrichtig'... zunehmend ihre klaren Konturen...“. Es werden „diskursive Wirklichkeiten geschaffen, die kein Abbild vorgängiger Tatsachen oder psychischer Zustände sind“. Ein konstruktivistisches Verständnis psychotherapeutischen Handelns stellt die „psychische Wahrheit" der Erzählungen als die für die Therapeut-Patient-Dyade verbindliche Konstruktion von Lebenswirklichkeit ins Zentrum. So findet der Erzähler in der Psychotherapie Resonanz für seine mentale Verfassung. Wenn Therapeuten sich für die Verbesserung der inneren und äußeren Lebenssituation der Patienten einsetzen, so geht es im Kontext der "diskursiven Wahrheit“ um eine verträgliche, nützliche, komfortable Umgestaltung potentiell maligner Wirklichkeitskonstruktionen, mit dem Ziel, Bildern der Depression, der Angst und anderer innerer Qualen den Zugang zu verschließen. Die Frage nach der „Zuverlässigkeit“ von Erzählern und Erzähltem, die in der Literaturwissenschaft seit Wayne Booth kontrovers diskutiert und konzeptuell weiterentwickelt wurde (Fludernik 2005; A. Nünning 1999; V. Nünning 2010; Zerweck 2004), hat somit zunächst sekundären Stellenwert, jedenfalls, wenn es nicht um pathologisches Lügen, paranoide Verdächtigungsnarrative oder strafrechtlich relevante Ereignisdarstellungen geht. 
Das heißt aber nicht, dass die Unterscheidung zwischen Faktualität und Fiktionalisierung auf Dauer hinfällig wird. Es heißt nur, dass die narrative Kommunikation im biografisch-therapeutischen Kontext Zustimmung, glaubensbereite Akzeptanz, emotionale Partizipation und Empathie verlangt. Freud reagierte wie erwähnt mit Skepsis auf bestimmte Erzählungen seiner „Neurotica“, sie nivellierten, meinte er, das eigene Interesse, die eigene Initiative und den eigenen Akteurstatus zu stark. Bis heute gilt sein Verdacht als anstößig. Ob Freud bestimmte Patientinnen nicht ernst nahm und elterliche Verantwortungslosigkeit bagatellisierte, ist nicht aufgeklärt, auch sind die Erzählungen der jungen Frauen nicht bekannt. Eine Inspektion der therapeutischen Gespräche von damals ist nicht möglich. Wir wissen nicht, ob Freuds Skepsis Ausdruck kommunikativer Irritation war. In jedem Fall ist es problematisch, Erzählern kein Gehör und keinen Glauben zu schenken. Sie artikulieren zum einen ihr Darstellungsanliegen und verweisen zum anderen auf Faktenbezüge. Immer ist die Gefahr gegeben, dass der Hörer einen Faktenbezug zu Unrecht verwirft. So erstaunt die massive und anhaltende Kritik an Freuds Distanzierung von den narrativen Selbstthematisierungen seiner Patientinnen nicht, insbesondere seit der sexuelle Missbrauch an Kindern in Familien, Schulen, Heimen und kirchlichen Einrichtungen und seine beunruhigende Verbreitung in der öffentlichen Wahrnehmung präsent ist. Narrativer Glaubenskredit zeichnet sich durch folgende Aspekte aus:

- Wer dem Erzähler narrativen Glaubenskredit entgegenbringt, zeigt humanes Entgegenkommen.

- Wer dem Erzähler narrativen Glaubenskredit entgegenbringt, nimmt die Chance wahr, die Erfahrungsperspektive des Erzählers zu teilen.

- Wer dem Erzähler narrativen Glaubenskredit entgegenbringt, nimmt die Chance wahr, sich ein Bild vom Erzähler, seiner Motiv- und Abwehrdynamik zu machen.

- Wer sich der Darstellung des Erzählers - mag sein, mit verdecktem Vorbehalt anschließt, zeigt menschlichen Takt und macht sich auch ein Bild vom Erzähler.

- Wer sich der Darstellung des Erzählers nicht anschließen kann, unter anderem, weil er sie problematisch, unglaubwürdig, inkohärent oder wenig verständlich findet, reguliert Takt und Dezenz, wendet sich der psychischen Situation des Erzählers zu und versucht womöglich, jenseits der narrativen Kommunikation, Faktenbezüge zu klären.

- Erzähler werben um narrativen Glaubenskredit (Lejeune 1989).

- Wer die Glaubensbereitschaft des Hörers erreicht, gewinnt Wohlwollen für sein Darstellungsanliegen. Wer die Glaubensbereitschaft des Hörers verfehlt, bleibt mit seinem Darstellungsanliegen allein.

- Narrativer Glaubenskredit lässt sich missbrauchen, manipulieren und verwirken. 


\section{Faktualität, Fiktionalisierung und ein Empörungsnarrativ}

Erzählen unterscheidet sich vom Berichten unter anderem dadurch, dass es evokativen Charakter besitzt, dem Dort und Damals also plastische und wirksame Präsenz verleiht, und dass es, auf den Autor zurückverweisend, Erfahrung organisiert (Clandinin und Connelly 2000) und in persönlicher Perspektive zur Darstellung bringt. Es geht, mit Fludernik, um den Erfahrungsgehalt, um „experientality“ (Fludernik 2009, 2010). Der Erzähler evoziert Vorgefallenes mittels nachträglicher Regieführung. Er arrangiert mit Hilfe der Versetzungsregie eine Startdynamik, eröffnet für den Hörer einen Erwartungshorizont, bindet ihn ein in den Fortgang einer sequentiellen Entwicklung, die auf ein gutes oder weniger gutes Ende hin steuert. Der Hörer ist eingeladen, sich in das erzählte Dort und Damals zu versetzen. Der Erzähler will, dass man Anteil nimmt und seine Bewertungen teilt (Nünning 2011/2012: 91-93).

Das folgende Beispiel eines Empörungsnarrativs der Psychoanalysepatientin Amalie, vorgebracht in der 221. psychoanalytischen Sitzung, bringt das sinnfällig zur Anschauung. Amalie (118. Erzählung, 221. Therapiesitzung, Zürcher Erzählarchiv Jakob, Original Ulmer Textbank $)^{1}$ :

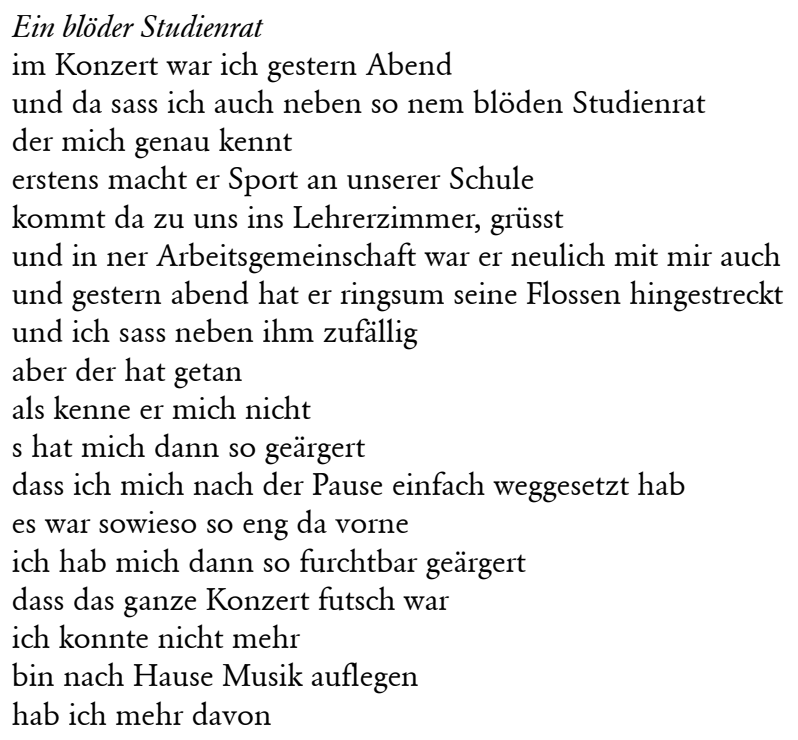

Gleich anfangs entwertet Amalie im Duktus kindlich-erregten Schimpfens den Sitznachbarn im Konzert mit dem abschätzigen Attribut blöd. Sie disqualifiziert

1 Zugunsten der Lesbarkeit verzichten wir bei diesem Transkriptausschnitt sowie den folgenden Transkriptausschnitten auf die Wiedergabe der regulären Transkriptionszeichen. Bei allen Transkriptwiedergaben sind die Namen geändert. Wortverkürzungen oder Wortentstellungen sowie fragmentarische Formulierungen werden dem Original gemäß beibehalten. Das Beispiel aus der Therapie von Amalie und das Beispiel aus dem Erstgespräch mit der Patientin Martha finden sich auch in Boothe (2015). 
den Kollegen mit einer wiederum abschätzigen Fisch-Bildlichkeit: gestern abend hat er ringsum seine Flossen bingestreckt, nur Amalie hat er nicht begrüßt. Er ist desinteressiert und ignoriert Amalie auf brüskierende Weise. Selbstherrlich streckt er ringsum seine Flossen hin, um gleichsam aus souveräner Haltung Audienz zu geben; auch hier dient die abschätzig kindlich flapsige Tierbildlichkeit der Entwertung und Verkleinerung der Lehrer-Autorität zum dummen Jungen mit gravitätischen Allüren.

Amalie wendet sich mit dem disqualifizierenden Porträt, das den Einfluss kindlicher Mentalität sinnfällig veranschaulicht, an ihr therapeutisches Gegenüber, in der Erwartung von Wohlwollen, Resonanz und sympathisierender Parteilichkeit. Der Erzählerin gilt die narrative Darbietung mit ihren von kindlicher Empörung getragenen Prägnanz- und Karikatureffekten als authentisch. Sie verlangt emotionale Partizipation an ihrer und Glauben für ihre biografische Ereignisschilderung. Im Alltag des mündlichen Erzählens äußert sich die wohlwollende Zuhörerschaft gewöhnlich mit Signalen und Kommentaren affirmativer Bestätigung. Gerade Erzählungen mit erregter Skandalisierungsrhetorik fordern kommunikative Resonanz. Der Analytiker greift hier den erregten Erzählduktus auf: Sie sind auch jetzt noch aufgebracht. Sodann leitet er einen wichtigen Schritt ein, der von einer Resonanz für die persönliche Perspektive des Erzählers zur Herausforderung führt, gemeinsam im Prozess der Selbstexploration und Reflexion den Anlass für die Erregung und Entrüstung zu untersuchen. Er sagt: Hat der Kollege Sie bezwusst ignoriert? Oder hat er Sie vielleicht nicht bemerkt? Die Patientin soll hellsichtig werden für ihr Erzählen als Konstruktions- und Bewertungsprozess, der auf konfliktdynamische Kompromisse und auf Abwehr im Dienst des psychischen Komforts verweist. „Als Abwehr bezeichnet die Psychoanalyse jede psychische Aktivität, die darauf abzielt, psychischen Schmerz in all seinen möglichen Formen zu vermeiden." (Müller-Pozzi 2002: 20). Eine Wahrnehmung, Erinnerung oder ein Handlungsimpuls verfällt der Abwehr, weil die bewusste Konfrontation damit Unlust bereitet. Die Abwehrmaßnahmen dienen der Aufrechterhaltung oder Wiederherstellung mentalen, psychosozialen und psychophysischen Funktionierens und dem vorübergehenden Schutz vor aversiven Reizen. Abwehr - kunstvoll ins Erzählen integriert, Fiktionalisierungsprozesse im Dienst psychischen Komforts begünstigend - entlastet kurzfristig, mindert aber erfolgreiche Selbst- und Situationsexploration und beseitigt die Konfliktspannung nicht. So entkommt Amalie vorübergehend der womöglich verletzenden Konfrontation mit der Zurücksetzung und Indifferenz mit Hilfe der Abwehrmaßnahme der Entwertung des blöden Studienrats und der Abwehrmaßnahme der Rationalisierung, zuhause habe sie mehr Musikgenuss.

\section{Der Faktenbezug ist fragreürdig - Biografisches Erzäblen unter Verdacht}

Wenn die psychotherapeutische Kooperation auf Dauer gelingt, werden die Narrative allmählich selbst zum Thema, dann gewinnt der Erzähler allmählich die Stärke, sich nicht nur dem Dargestellten zu widmen, dem vermeintlich Fakti- 
schen, sondern auch dem gerade auch in Fiktionalisierungsleistungen wirksamen Darstellungsanliegen, dem, was er aktualisierend, um Resonanz werbend, bewältigungs- und wunscherfüllungsorientiert erreichen will. Es wird im gemeinsamen Prozess des dekonstruktiven Erkundens der narrativen Inszenierungsmuster immer deutlicher, in welcher Weise die Erzählung vor allem auf den Autor zurückverweist, auch wenn sie diesem zunächst vor allem als valide Schilderung seiner Lebenswirklichkeit gegolten hatte.

Es gibt auch prekäre Erzählungen, deren Faktenbezug Hörern unmittelbar als problematisch gilt. Psychiater, Psychotherapeuten und psychologische, seelsorgerische oder juristische Berater kennen Patienten und Ratsuchende, deren Erzählen das Gegenüber nicht zu empathischem Engagement einlädt, sondern distanzierende Reaktionen wie Skepsis, Misstrauen oder Unglauben provoziert. Hier steht das Erzählen unter Verdacht. Das gilt traditionell für Erzählungen von Personen, die man als paranoid einschätzt. Man hält ihre Erzählungen, in denen sie verfolgt, belauert und infam geschädigt werden, für so unwahrscheinlich, dass sie keinen Glauben finden. Erzählungen von Personen, deren Erfahrungen in hohem Maß erwartungswidrig sind, werden als wahnhaft, als Realitätsverkennungen eingeschätzt. Erzählungen von außergewöhnlichen Erfahrungen, etwa ungewöhnlich frühe Kindheitserinnerungen, wecken Skepsis; hier besteht hoher Plausibilisierungsbedarf, oder sie werden verworfen. Spektakuläre biografische Erzählungen mögen in hohem Maße beeindrucken, Erzählungen mit ausgeprägt sieghafter und prunkhafter Ich-Profilierung mögen amüsieren, aber sie setzen sich kritischer Beurteilung aus, was die Redlichkeit der Darstellung angeht. Personen, die andere des Missbrauchs, der Gewalt und maligner Manipulationen bezichtigen, fordern Glauben, finden empörte Parteinahme, laufen andererseits aber auch Gefahr, absichtlicher Falschbezichtigung verdächtigt zu werden². Die Beispiele zeigen: Erzählen steht hier unter Verdacht. Psychiater und Psychotherapeuten erhalten in den Erzählungen Aufschluss über die mentale Welt der Patienten $^{3}$, doch anders als bei unauffälligen Narrativen gerät die Frage nach der Wahrheitstreue und Tatsachengerechtigkeit in den Vordergrund ${ }^{4}$.

\section{Eine Patientin erzäblt - die Therapeutin ist irritiert}

Im Folgenden geht es um ein Erstgespräch, das es dem therapeutischen Gegenüber schwer machte, (1) sich ein erstes Bild von der Problematik zu machen und angemessen mitzuteilen, (2) eine Offerte der Zusammenarbeit, zugeschnitten auf

2 Zur Befundlage der empirischen Glaubwürdigkeitsforschung Nawratil (2006).

3 Zum paranoiden Erzählen Freuds (1911) eindrucksvolle und prominente Analyse der Biografie eines paranoiden Mannes.

4 Auch ausgeprägte Expertise und lange Berufserfahrung, exemplarisch im Bereich der juristisch und administrativ relevanten Ereignisrekonstruktion, bleiben der Fehleinschätzung ausgesetzt (Nawratil 2006: 217-220). 
die spezifische Patientin-Therapeutin-Dyade, zu formulieren, (3) im narrativen Prozess am emotionalen Mitvollzug zu partizipieren und Gelegenheit zum analytischen Erschließen zu finden. Patientin und Therapeutin bestimmten dennoch am Ende der Erstkonsultation einen zweiten Termin. Die Therapeutin machte auch Vorschläge, was bei diesem zweiten Termin zur Sprache kommen könnte, vor allem die Biografie der Patientin und die Geschichte der Beziehung zum Ehepartner. Die Patientin sagte das vereinbarte Gespräch zu einem späteren Zeitpunkt ab, ohne die Absage näher zu begründen.

So kann auch die im Folgenden vorgestellte Untersuchung in Bezug auf die psychische Situation der Erzählerin keinen sicheren Ertrag bringen, jedoch punktuell klären, wie insbesondere die narrative Selbstthematisierung der Ratsuchenden zu Erschwernissen und Beeinträchtigungen hinsichtlich der Verständigung, der Verdeutlichung von Akzeptanz und der Ermutigung zur Auseinandersetzung führen kann. Die sechzigjährige Frau mit dem Decknamen Martha konsultierte die psychodynamisch orientierte Praxisstelle des Psychologischen Instituts der Universität, weil sie sich in einer krisenhaften Situation befand. Es war der Patientin (im Folgenden P) sehr wichtig, einen Ereigniszusammenhang zu schildern, der für ihre aktuelle psychische Belastung aus ihrer Sicht von entscheidender Bedeutung war. Die Erzählerin betonte den außergewöhnlichen und befremdlichen Charakter der Geschehnisse; die langen und verwickelten Ausführungen gestalteten sich als nachträgliche Selbstvergewisserung hinsichtlich dessen, was sich zugetragen hatte, was sie aus erster oder zweiter Hand wusste oder eben nicht wusste. Die Therapeutin (im Folgenden T) reagierte mit dezenten Zeichen der Irritation und Distanzierung; sie begegnete den expansiven Rede- und Erzählsequenzen der Ratsuchenden in reservierter Haltung und fragender Distanz. In der Kommunikation der Beteiligten etablierte sich keine „Handlungskoordination“ im Sinne der Einstimmung auf und Realisierung des gemeinsamen Gesprächsunternehmens ,psychotherapeutisches Erstgespräch' als joint project (Deppermann 2007: 8).

\section{Die Initialphase}

$\mathrm{Zu}$ Beginn verständigen sich Therapeutin und Patientin über finanzielle und organisatorische Belange; $\mathrm{P}$ ist problemlos einverstanden. Die Therapeutin positioniert sich initial als Regieführerin in der Gesprächsorganisation. Sie bittet die Patientin, die für die Therapeutin noch nicht verständliche, bei der Anmeldung im Sekretariat niedergelegte Botschaft auf dem Anmeldeformular der Institution zu erläutern. und zitiert wörtlich die Notiz der Sekretärin: ${ }^{5}$

T: so und jetzt - wollen wir mal auf Sie? zu sprechen? kommen? - und zwar ähm, haben Sie ja mit der Sekretärin? geredet?P: hmhm

5 Fragezeichen markiert Stimmhebung mit Frageintonation; Komma markiert einfache Stimmhebung; Ausrufezeichen markiert Betonung. 
T: und haben dort, gesagt also ich wir haben so ein kleines Formular?

P: Sie haben

T: und so ein bisschen darauf geschrieben wird wieso Sie sich an unsere

P: hmhm

T: Stelle? wenden und dort

P: hmhm

$\mathrm{T}$ : ist gest- dort drauf ist gestanden dass Sie: ähm, besorgt! seien weil sich ein totgeglaub-

ter Bekannter von Ihrem Mann?

P: hmhm

T: bei Ihnen in der Wohnung eingenistet hätte

P: ja so ungefähr kann man das sagen

$\mathrm{T}$ : hmhm tönt (umgangssprachlich für „klingt“) für mich? noch nicht ganz verständlich?

P: ja:? es ist

T: und darum wäre ich froh? wenn Sie es mir ein bisschen ausführen könnten.

Die Therapeutin äußert unmittelbar zu Beginn ein Nicht-Verstehen, fordert zum Ausführen auf. Damit ist für Martha ein verbindlicher Bezugsrahmen etabliert. Sie ist auf die Formulierung festgelegt und soll ihr durch Erläuterung Evidenz verschaffen. Martha quittiert den Auftrag, hebt an mit einem betonten, längere Ausführungen ankündigenden summarischen Partikel (also!), versichert ihrem Gegenüber, dass sie dem Auftrag nachzukommen vermag, und zwar weil die Angelegenheit, um die es geht, sie aufs Äußerste bedränge und beherrsche. Was sich ihrer bemächtigt hat, das hat ein genaues Datum, den 1. Juli, dort kam es - wie es kam, bleibt zunächst offen - zu einer gänzlichen Umwälzung ihres Lebens. Betont werden Dramatik und Folgenreichtum dessen, was sich an und mit Martha vollzogen hat. Der Verursacher ist jene männliche Person, die als totgeglaubter Bekannter des Ehemannes zitiert wurde. Die Therapeutin äußert, das Mitgeteilte sei für mich? noch nicht ganz verständlich? Sie sagt nicht: Das klingt ja interessant, Sie machen mich neugierig! Dies wäre eine Option, auf den Lockruf- oder Schlagzeilencharakter der Formulierung vom totgeglaubten Eingenisteten zu reagieren und ein eigenes Fasziniertsein kundzutun, das für die Klatschkommunikation (Bergmann 1987) charakteristisch wäre.

\section{Ein gefährlicher Mann}

Max, der Antagonist, wird eingeführt als Persönlichkeit von öffentlichem Interesse, als angesehener, wohlsituierter Mann, der zum wohnungslosen Flüchtling vor Polizei und Gläubigern wurde. Martha stellt im Folgenden die Beziehung zwischen ihrem Ehemann und dem journalistisch prominenten Max dar: Es habe lose, wenn auch kontinuierliche und branchenübliche Berufskontakte gegeben. Ihre eigene Beziehung zu Max wird als flüchtig und nur über den Ehemann vermittelt dargestellt. Zugleich konzediert sie gelegentliche private Treffen und informelle Begegnungen, auch der beiden Ehepaare, man sagt einander Du; das ändere aber am marginalen und indifferenten Charakter der Beziehung nichts. In diese belanglose Indifferenz hinein fährt die Sensationsnachricht vom unange- 
kündigten Verschwinden des Max. Was es damit im Einzelnen auf sich hatte, erfährt P nur über Dritte, auch über den Ehemann, manchmal im Nachhinein oder aus der Zeitung: Max als mittelloser, delinquenter Bankrotteur, vor der internationalen Polizeifahndung flüchtig, Kollegen, auch den Ehemann, um Geld anbettelnd, eine der P bis dato unbekannte Kollegin gewährt dem Wohnungslosen Unterschlupf, auch von dort verschwindet er unangekündigt. In szenischen Vignetten gibt P Dialogpassagen im Duktus der wörtlichen Rede mit dem Ehemann wieder. Stets eröffnet der Ehemann als Redner den kurzen Dialog, eine neueste Meldung über Max kolportierend. Stets endet die szenische Vignette mit der Versicherung, dass P die Angelegenheit desinteressiert fallen lasse. Auch indem $P$ vorführt, wie sie sich auf gewisse Namen und Verhältnisse erst besinnen müsse, vermittelt sie Distanz zum einstigen Geschehen, dem P sich nicht von sich aus hatte nähern wollen, sondern das ihr aufgedrängt wurde. $\mathrm{P}$ positioniert sich als Darstellerin der Entwicklung des Geschehens in unbeteiligter Außenperspektive, markiert durch fortlaufende Versicherungen eigener Indifferenz: und da hab ich mich gar nicht weiter darum gekümmert? und und! äh, ich habe mich dann weiter? auch? nicht? da- der Fall war für mich erledigt? - Die unbeteiligte Außenperspektive würde den Rededuktus knapper summarischer Sachinformation erwarten lassen. Doch die szenischen Vignetten, die dramatischen Wiederholungen des Rufs Max ist verschwunden! und die detaillierte Wiedergabe etwa des von Max verursachten Problems mit Tanjas Wohnungsschlüssel vermitteln kommunikativ einen gegenteiligen Eindruck: Die Erzählerin präsentiert sich keineswegs indifferent oder in kühlem Abstand, sondern als hoch involviert, stets auf neue Botschaften ansprechbar, wenn auch unzureichend und widersprüchlich informiert.

\section{Martha und die Skandaltriade}

Die Versicherung von Desinteresse und Indifferenz kollidiert überdies mit dem Duktus der skandalierenden Rede, der das gesamte Erstgespräch bestimmt. Martha ist ohne eigenes Zutun von einem Ärgernis betroffen, das öffentliches Aufsehen erregt. Die Schlüsselfigur ist in eine juristisch und wirtschaftlich relevante und zugleich undurchsichtige Affäre verstrickt, die ihre Reputation beschädigt und sie moralisch disqualifiziert. Martha bringt diesen Skandal im Erstgespräch zur Darstellung. Dabei kommt es zur Inszenierung einer kommunikativen Situation, die Nawratil, mit Neckel (1986), als „Skandaltriade“ anspricht: „...der Skandalierte (der einer Verfehlung von öffentlichem Interesse öffentlich bezichtigt wird), der Skandalierer (einer, der diese Verfehlung öffentlich denunziert) sowie ein, oder besser: mehrere Dritte, denen über das, was zum Skandal geworden ist, berichtet wird und die daraufhin eine wie auch immer geartete Reaktion zeigen" (Neckel 1986: 585). Neckel und Nawratil geht es bei diesen Ausführungen um den politischen Skandal, die Trias lässt sich jedoch mühelos auf den sozialen Nahraum übertragen. Martha führt den Antagonisten Max als Skandalierten ein, 
betätigt sich selbst als Skandaliererin und sucht, ihr Gegenüber zu einer der Skandalisierung zustimmenden Reaktion zu bewegen. Dabei setzt sie „selektive Definitionsmacht" (Nawratil 2006: 204) ein, das heißt, sie macht im Darstellungsprozess die eigene normative Bewertungsperspektive geltend, unter Vernachlässigung anderer möglicher Deutungshorizonte. Inhalte, die „die eigene Sichtweise stützen“, werden hochgespielt, zuungunsten solcher, „die der eigenen Sichtweise zuwiderlaufen.“ (210). In solchen Darstellungen haben „Akteure, die eine andere Sicht der Dinge vertreten, nur geringe Chancen, ihre Position unverfälscht zu präsentieren. Stattdessen wird über sie nur bruchstückhaft und wertend berichtet.“" (210). Das wird bei Martha sehr sinnfällig. Dialogpartien mit wörtlichen Redezitaten - Martha und ihr Ehemann, Martha und Max - sind überaus häufig. Stets profiliert sie die eigenen Redebeiträge als denjenigen der Gesprächspartner überlegene. Generell geht Martha „offensiv“ vor, indem sie „von der eigenen Seite ein möglichst positives Bild“ vermittelt und „dem Gegner negative Eigenschaften“ (211) zuschreibt. Sie stellt - auch dies charakteristische taktische Mittel - Kompetenz und Glaubwürdigkeit des Skandalierten infrage. Sie kontrastiert dies mit der eigenen Selbstdarstellung als vertrauenswürdig und dominiert darüber hinaus das Gespräch (212). Marthas Rededuktus ist expandierend, dramatisch drängend, gekennzeichnet durch kontinuierliche Beibehaltung des Redezugs. Das wird erreicht durch das Fehlen von Sprechpausen, Stimmhebung am Ende von Äußerungen und Äußerungspartien, szenischen Vignetten, Dialogzitaten, detaillierten Beschreibungen, selbstinitiierten evaluativen Kommentierungen vorausgegangener Mitteilungen, schwach markierten Übergängen zu episodischen Narrativen, in die Martha mehrfach längere Beschreibungen und Kommentare einflicht, bevor sie dann die narrative Entwicklung wieder aufnimmt. Angebote der Redeübergabe an ihr Gegenüber bleiben aus. Ausdrückliche interaktive Offerten wie Fragen oder Bitten fehlen; ebenso fehlen Zäsuren als schweigendes Innehalten im Mitteilungsfluss; es gibt keine Reduktion des Sprechtempos, etwa, um in verlangsamtem Sprechtempo, im explorativen oder reflexiven Modus zu kommunizieren.

\section{Martha als unzuverlässige Erzäblerin}

Marthas episodisches Erzählen weist Charakteristika der unzuverlässigen Narration auf (V. Nünning 1999): Der Hörer ist darauf eingestellt, der narrativen Regieführung der Sprecherin zu folgen und sich in ein Dort und Damals zu versetzen. Die narrative Ereigniskonstruktion startet. Die Hörerin ist auf Mitvollzug eingestellt, die Bühnenregie führt aber vom Geschehen weg zu weitläufigen Beschreibungen mit unbestimmtem Ziel, beleuchtet dann wieder szenisches Geschehen, dessen Anschluss an das Vorangegangene allerdings im Ungefähren bleibt. So ist die Hörerin auf Orientierungssuche mit unsicherem Erfolg und mit ungenügenden Orientierungshilfen, dabei dem kontinuierlich fortlaufenden Re- 
defluss ausgesetzt, der neuen, dramatischen Brennpunkten zustrebt. Es kommt zu nach Orientierung suchenden Nachfragen mit eingeschränktem Klärungserfolg. So tritt an die Stelle der partizipativen, emotional involvierten Imagination beim Hörer Irritation und Distanznahme. Die Chance, eine Erzählung zum Gegenstand gemeinsamer Exploration und Reflexion zu machen, eröffnet sich nicht, da fehlende Abschlussmarkierungen die narrative Dynamik oft in der Schwebe lassen, und somit das Rederecht weiter bei der Erzählerin bleibt, die übergangslos neues Material präsentiert. Ob ein massives unterbrechendes Eingreifen der Therapeutin das kommunikative Zusammenspiel verändert hätte, muss offen bleiben.

\section{Der gefährliche Mann sucht Unterschlupf}

Ein eindrucksvolles narratives Beispiel wird exemplarisch untersucht; es ist aus dem Redekontext, der selbst narrativ organisiert ist, herausgeschnitten. Die Transkriptpassage wird vorgestellt, in der Martha schildert, wie Max sich nach langer und geheimnisumwitterter Abwesenheit unangemeldet an ihrer Wohnungstür präsentiert. Es handelt sich um eine Erzählung mit expansiven Vorschaltungen und der anschließenden Darstellung des überraschenden Besuchs von Max in der Privatwohnung des Ehepaares: Max wird zum vorübergehenden Verbleib eingeladen, gepflegt, versorgt und zum geselligen Beisammensein eingeladen.

P: sind, jetzt? ist das ein Jahr, im Juli ist ein Jahr sind wir von der A-Strasse in die BStrasse gezogen.

T: ja hmhm

P: und wir wohnen im Sous-sol? unten? und jetzt haben wir Gegensprechanlage aber ich musste immer? ich konnte entweder aufdrücken? oder ich bin immer rauf gegangen und hab aufgemacht damit ich damit nicht einfach jeder reinkommen, kann oder?

T: genau hmhm hmhm+

P: und weil das ist mir ein paar Mal passiert? auch die Kinder oben dann läuten sie und ich drück auf? und auch ja? je nachdem wer ist

T: hmhm

P: und ähm, am ersten! Juli, mein Mann kommt nach Hause wir waren eingeladen? im Aargau? und hat er gesagt ich komm früh? nach Hause wir gehen dass wir um fünf halb sechs da? sind?, zu einer alten, Tante die ist schon, fünfundsiebzig und sie freut sich immer wenn jemand kommt und wir gehen sie $a b$ und zu besuchen

T: hmhm

P: da hatten wir abgemacht? dann hat sie gesagt „ja! sie macht den Grill? im Garten an“ da hab ich gesagt „ja? ich bring Fleisch und so wie das ist“. kommt mein Mann ungefähr um zwei nach Hause? hat gesagt eben? er macht „noch das und das? und dann gehen wir früh? wir haben ungefähr eine Stunde? und: dann kommen wir auch wieder früh nach Hause“ - der ist keine halbe Stunde da da läutet es, dann habe ich gesagt „hm sind doch sicher? wieder Kinder oben oder?", und dann geh ich da rauf? und dann sehe ich da durch die Glasscheibe einen Mann? mit einem Rucksack? oder? dann hab ich gedacht ja das gehört zu den Leuten? da oben? wieder oder?

$\mathrm{T}: \mathrm{hmhm}$ 
P: und: ähm, dann mach ich auf?, steht? doch dieser Mensch vor mir, sie glauben! es nicht, ich bin fast, ich musste mich festhalten? mir ist schwarz vor Augen geworden?

T: hmhm hmhm

P: und wie der Mensch aussah? ich mein klar? er, er kann sich nicht rasieren und nicht; aber Bart! lange Haare, alles, ja, dreckig? wirklich:, ich hab mich festgehalten? an der Tür ich hab gesagt „mir wird's schwarz! vor Augen?“ sag ich „wo kommst denn du? her!“

T: hmhm

P: er so „ja, bin jetzt da?“ dann sag ich ,ja komm rein?!“ oder? - ich mein Mann gerufen? „Peter! Peter! komm?!“ oder? und er natürlich ein „Hallo:?! ja wo kommst du her?!“ und so und: dann hab ich gesagt ,ja setzt euch?“ und äh „ihr müsst was trinken?“ und so. und dann äh, hat er gesagt ,ja?“ da sag ich „ja, wieso hast du nicht telefoniert? oder, eine Karte? geschrieben / oder dich mal, gemeldet?“ oder, da sagt er „ja weisst du, es soll niemand wissen dass ich da bin"

$\mathrm{T}: \mathrm{hmhm}$

P: hm? und dann, hab ich gesagt ,ja, also komm? zieh dich aus?“ oder? und äh ja sagt sagt mein Mann „ja wo schläfst? denn du?" sagt er „ja“; jetzt die letzte? Nacht er hat so ein kleines Zelt hat er auf der Rigi! oben geschlafen

$\mathrm{T}: \mathrm{hmhm}$

P: noch im Wald oder +wo wo auch immer

$\mathrm{T}: \mathrm{hmhm+}$

P: und dann äh, mein Mann gleich ,jaja du kannst bei uns! wir haben eine grosse Wohnung? und äh hm, das geht schon?" und äh dann hab ich gesagt ,ja also, das geht schon? ja wir können das wenn ich Besuch habe wie so ein Notbett und im Wohnzimmer haben wir gleich das Bett?!“ oder / ähm er kann er hat schon dann hm Dusche und WC das kann er vorne benutzen? und ähm, dann äh a- haben wir das eingerichtet?! und äh, weil mein Mann sagt „ich bin ja so! froh dass du das bist und dass du dich ja wir haben immer! v- ja also nicht immer aber oft!? von dir geredet und eben das letzte was wir gehört haben, du bist auf dem Pilgerweg gesehen worden.“ und: dann äh sagt er ,jaja? das ist schon möglich" weil er hat in Frankreich?, hat er damals in einer Kirche auch gepredigt? und und gesprochen und so und das ist so üblich? mit den Pilgern weil ich hab mich jetzt, ein bisschen mit dem ge- befasst?

$\mathrm{T}: \mathrm{hmhm}$

P: mit der Pilgerei - also intensiver als vorher

$\mathrm{T}: \mathrm{hmhm}$

P: und dann? - äh, ja also was machen wir jetzt heute Abend sind wir eingeladen haben wir gesagt ,ja also zu Daniela das ist, im Garten! das ist unkom- und sie ist unkompliziert oder? da können wir ihn ruhig mitnehmen“ ich telefoniert? und hab gesagt „du macht das dir was aus?“ und so sagt sie „nein nein! das ist egal im Garten da könnt ihr noch zwei drei Leute mitbringen das ist mir egal“

T: hmhm

P: dann haben wir den Mann! erst mal, äh, drei! Mistkübelsäcke hab ich Abfall, er hat alle alles ja weggeschmissen das hat ja gestunken?

$\mathrm{T}: \mathrm{hmhm}$

P: ist wirklich! grusig (umgangssprachlich für widerlich) gut, es ist mir auch noch egal also auf jeden Fall drei! Mistkübelsäcke voll dann haben wir ihn erstmal unter die Dusche? hier hast du dusch- / erst mal. Unterwäsche? und von meinem Mann Hemd und alles äh hervorgesucht oder? damit er hatte? ja, ja ich konnte es ja auch? nicht? gleich? waschen?! oder?

$\mathrm{T}: \mathrm{hmhm} \mathrm{hmhm}$ 
P: auf jeden Fall ha- hat er haben wir ihn dann erst mal so? versorgt, und dann, haben wir ihn da mitgenommen an dem Abend und äh, ja? ich mein er hat dann schon ein bisschen so erzählt? er hat aber nie:? so sehr viel erzählt...

Auf das Läuten hin rechnet die Ich-Figur mit einem Störer oder einem Fremden, der eine andere Adresse sucht. Doch der Mann mit Rucksack ist überraschenderweise Max. Der Anblick des Mannes, wild und entblößt von zivilisierter Gepflegtheit, lässt Martha geradezu den Boden unter den Füßen verlieren. Offen ist, was im Einzelnen die Ursache der Gefahr des Gleichgewichtsverlustes - ich musste mich festhalten - war. Marthas Frage wo kommst denn du? her! bleibt unbeantwortet. Max macht lakonisch geltend ja, bin jetzt da? und erhält ein festliches Entrée: dann hab ich gesagt ,ja setzt euch?“ und äh „ibr müsst was trinken?. Der Hinweis auf seine Wohnungslosigkeit und die Eröffnung, dass sein Aufenthaltsort geheim zu halten sei, macht den Besucher zum Asyl Suchenden. Die Gastgeber, nunmehr Mitwisser, voran Martha als Akteurin, zeigen voll Eifer, dass sie Asyl gewähren wollen. Und nicht nur das: und dann, hab ich gesagt ,ja, also komm? zieh dich aus?" Dezenzregeln werden gelockert zugunsten der Re-Zivilisierung des Gastes. Seine Mittellosigkeit und Angewiesenheit auf Zuflucht und Versteck reduzieren Max jedoch nicht auf den Status des Bettlers. Im Gegenteil: mein Mann sagt „ich bin ja so! frob dass du das bist und dass du dich ja wir haben immer! v-ja also nicht immer aber oft!? von dir geredet. Martha kündigt Max für das familiäre Treffen als Gast an. Dort tritt er in Begleitung des Ehepaares in gesitteter Ausstattung auf.

\section{Schauder, Faszinosum und Begehren}

Max tritt ins Gesichtsfeld der Ich-Figur als Erscheinung im Spektrum von Schauder und Faszinosum, die unmittelbar und imperativ ihre Präsenz geltend macht bin jetzt da. Max ist willkommen und wird festlich geehrt. Durch Marthas Engagement verwandelt sich die wilde in eine zivilisierte Erscheinung. Der Gast nutzt das neue Zuhause des Ehepaares als Zuflucht. Die Erzählung liefert keinen Hinweis darauf, dass er die beiden Helfer seinerseits schätzt und würdigt; diese indessen begrüßen und behandeln ihn so, als ob er dem Haus zur Ehre gereiche. Die Ankunft des Vermissten wird auch im Bezug nach außen, der Gartengeselligkeit bei der Tante, gefeiert.

Die Erzählung zeigt Marthas Beziehung zu Max in neuem Licht. In der Erzählung verhält sich Martha Max gegenüber in keiner Weise indifferent. Im Gegenteil, die Erscheinung des Mannes haut sie geradezu um. Allerdings, ohne sie sprachlos zu machen. Sie ist engagierte Initiatorin, Akteurin und Arrangeurin. Max hat Marthas Zuhause als diskreten (niemand soll das wissen) Ort der Zuflucht gewählt. Dies wird mit Freude und gastlicher Dienstbarkeit begrüßt, als sei es eine Auszeichnung und ein Privileg, Max beherbergen zu dürfen. Die Welt soll davon erfahren. Diskretion wird nicht gewahrt. Immer haben die Eheleute von Max gesprochen, heißt es in der Erzählung. Zwar folgt gleich darauf eine Rede- 
korrektur hin zu nicht immer, aber oft. Jedoch steht das in deutlichem Kontrast zu den vorangegangenen zahlreichen Indifferenzbeteuerungen. Die Korrektur hin zu nicht immer, aber oft soll den Kontrast mildern, macht aber umso deutlicher auf ihn aufmerksam. Die Erzählung ist effektvoll, hat aber diverse Plausibilitätsprobleme. Max wird ständig gesehen, muss sich aber verstecken. Wie passt das zusammen? Max muss sich verstecken, wird aber zum geselligen Anlass mitgenommen. Wie passt das zusammen? Max kommt nur mit Rucksack, der zu beseitigende Müll füllt aber mehrere Säcke. Wie passt das zusammen?

Dass die Erzählung Neues in Bezug auf Marthas mögliche psychische Belastung zum Vorschein bringt, ist deutlich. Abwehrmaßnahmen wie Verleugnung und Verneinung werden sinnfällig: Es geht um den dysfunktionalen Versuch, ein mächtiges Affiziertsein von einer prominent profilierten und zugleich die bürgerliche Ordnung sabotierenden Figur, die jedermann zu faszinieren scheint, abzuwehren. Es würde darum gehen, der Abwehr und ihrer Motivierung in der therapeutischen Arbeit auf die Spur zu kommen. Marthas Ausführungen stehen im Bezugsrahmen der Aufforderung, ihr Anliegen an ein therapeutisches Gegenüber verständlich zu machen. Die Therapeutin aber wird nicht zur engagierten Dritten in der Skandaltriade, sondern verbleibt in reservierter Distanz. Martha versichert, weder Sensations-, noch Skandal- noch Klatschakteurin zu sein. Vielmehr drängt sich ihr, ohne eigenes Zutun, ein Geschehen auf, das sich ihrer unheilvoll bemächtigt, wie sie im letzten Teil des Gesprächs zum Ausdruck bringt. Diese unheilvolle und leidvolle Bemächtigung hat sie ihres Seelenfriedens und der harmonischen Beziehung zum Ehemann beraubt - so thematisiert sie am Ende ihr auf Psychotherapie zielendes Problem. Die Therapeutin macht den Vorschlag, an dieses Problem in einer zweiten Sitzung anzuknüpfen. Die Patientin stimmt vorbehaltlos zu, doch nimmt sie dann die Gelegenheit nicht wahr und meldet sich nicht mehr.

\section{Faktualität und Fiktionalisierung im narrativen Kontext: Aufgaben des Therapeuten}

Eigene Präferenzen und Relevanzen werden im Erzählen wirksam. Die narrative Inszenierung von Ereignissen hat für den Erzähler Aktualisierungs-, sozial integrative, wunschorientierte und Bewältigungsfunktion (Boothe 2011). Martha $a k$ tualisiert erregt die Geschichte einer Begegnung, die sie nicht hinter sich lassen kann. Sie präsentiert sich im Dienst sozialer Integration vor ihrer psychoanalytischen Zuhörerin in souveräner Indifferenz, aber auch als hilfsbereite Gastgeberin. Die Präsentation leidet freilich unter Plausibilitätsmängeln, allzu deutlich drängen Wunschmotive, die keine bewusste Akzeptanz finden, vermutlich im Bereich des erotisch-Sexuellen, in den Vordergrund; die kontinuierliche Versicherung des eigenen Desinteresses als Abwehrstrategie gibt diesem Hörer-Eindruck Nahrung. Martha ringt um Form, Kontur und um eine Darstellung, die ihr nachträglich Regieführung und Überblick verschafft. Diese Bewältigungsleistung ist bisher nicht 
gelungen; um diese nachträgliche Bewältigung würde es in der gemeinsamen therapeutischen Arbeit gehen.

Allgemein formuliert:

- Erzählende Aktivität erlaubt, Vergangenes zu aktualisieren und auf diese Weise Anschluss an die gegenwärtige Situation zu finden. Die Trennung vom historisch Vergangenen wird durch die Herstellung von Präsenz in einer Beziehungsgegenwart aufgehoben.

- Erzählende modellieren eigene Identität vor dem sozialen Gegenüber, das heißt, sie positionieren sich mit ihrer narrativen Offerte im sozialen Kontext. Die Erzählung wendet sich an ein Gegenüber im Dienst der Herstellung sozialer Integration und konturierter Identität. Der Wunsch nach Anerkennung durch bedeutsame Andere und sozialer Integration fordert die erzählende Person zu einer narrativen Gestaltung heraus, die dem Glaubenskredit des wohlwollenden Publikums Rechnung trägt, ihn nicht strapaziert oder ausbeutet.

- Die narrative Dramaturgie modelliert Situationen nach einer spezifischen Wunscherfüllungstendenz. Das ist die dem Lustprinzip verpflichtete Optimierung. In diesem Sinne steht die Erzählung im Dienst eines Interesses an nachträglicher hedonischer Korrektur. Erzähltes Leben bewegt sich in der Spannung zwischen Erfüllung und Katastrophe. Dieser Prozess narrativer Verhäuslichung heißt, dass Lebenswirklichkeit gebaut und als Glücks- und Katastrophenmodell eingerichtet wird; als Ereigniszusammenhang, der sich zwischen dem Wünschbaren und dem Aversiven positioniert.

- Erzählen im Dienste der psychischen Stabilisierung oder der psychischen Reorganisation: Diese Modellierungsleistung ist eine Bewältigungsstrategie psychischer Destabilisierung, z. B. von erlittener Bedrohung oder negativer wie positiver Überraschung, mit der versucht wird, erlittene Erschütterung, psychische Destabilisierung in negativer, traumatisierender oder in positiver, euphorisierender Richtung im Nachhinein durch wiederholtes Erzählen zu integrieren. Der aktive Prozess des Gestaltens modelliert erregend-spannungsvolle und emotionalbewegende Situationen zu einem organisierten und kontrollierbaren Ganzen und führt zum Gewinn narrativer Regieführung und imaginativer Strukturierung.

Ein Patient, der psychotherapeutische Behandlung sucht, befindet sich in der Situation des Erstkontakts in einer prekären Lage. Angesichts seiner psychischen Belastung sieht er sich zur Kontaktaufnahme mit einer professionellen Helferinstanz genötigt und muss einem Fremden gegenüber gleichsam einen Offenbarungseid leisten. Für den Therapeuten geht es beim Erstkontakt darum, sich einzustellen auf die persönliche Perspektive des Ratsuchenden, auf die individuellen Bedingungen seines Leidens und seiner Konfliktdynamik. Das kann sich als anspruchsvolle Herausforderung erweisen (Hermann et al. 2012).

Bei der Ratsuchenden Martha gab es Hinweise auf die Konfliktdynamik, doch keine Chance, (1) die Eindrücke der Therapeutin kommunikativ zu vermitteln 
und kooperativ fruchtbar werden zu lassen, (2) eine Offerte zur Zusammenarbeit, zugeschnitten auf die spezifische Patientin-Therapeutin-Dyade, zu formulieren, (3) im narrativen Prozess am emotionalen Mitvollzug zu partizipieren und Gelegenheit zum gemeinsamen analytischen Erschließen zu finden.

Es versteht sich, dass die vielfältigen Anforderungen und Erwartungen an Therapeut und Patient Irritationen im Erstkontakt begünstigen. Die persönliche Perspektive, die ein Patient in seinen biografischen Schilderungen vermittelt, mag beim Gegenüber Befremden oder Abneigung anstelle von Wohlwollen und Empathie hervorrufen. In diesem Fall stehen Ratsuchender und Therapeut vor der Aufgabe, das Irritationspotential als Möglichkeitsraum zur Erschließung der Psychodynamik und Beziehungsdynamik zu nutzen (Rutishauser 2007). Narrative, die irritieren oder gar befremden, sind entscheidende Risikofaktoren für das $\mathrm{Zu}$ standekommen einer therapeutischen Bündnisbildung; sie konfrontieren das therapeutische Gegenüber mit hohen Ansprüchen an seine Fähigkeiten, den Ratsuchenden kommunikativ zu erreichen und ein für den Ratsuchenden aussichtsreiches Beziehungsangebot wirksam werden zu lassen, ein Beziehungsangebot, das die kommunikativen Schwierigkeiten zum klärungsfähigen und vielversprechenden Thema werden lässt (Grimmet 2014; Hermann et al. 2012).

Respekt, Wohlwollen und couragiertes Engagement, auch in befremdlichirritierenden Beziehungszusammenhängen, machen sich in besonderer Weise geltend: Der Therapeut engagiert sich für die Leidenssituation und das individuelle Patienten-Anliegen (Frei et al. 2012). Der Therapeut bemüht sich um Transparenz in der Verständigung, bei der Erläuterung des Behandlungsvorschlags und bei der Entscheidungsfindung. Dabei fordert er die Urteilskompetenz, Reflexivität und kritische Intelligenz des Patienten heraus. Er lässt seine persönliche und fachliche Autorität durch den Patienten testen und kritisch prüfen. Andererseits erwartet er vom Patienten, Mut zur Selbstoffenbarung aufzubringen. Es geht um eine Beziehung mit Vertrauens- und Zukunftskredit (Boothe und Grimmer 2005; Grimmer 2006; Mathys und Boucsein 2011). Die Vermittlung von Zuversicht durch den Therapeuten hängt von der rasch sich bildenden und oft unbewusst interaktiv wirksamen Einstellung zum Patienten ab (Streeck 2004). Diese Einstellung formt sich ab dem ersten Kontakt in „ein[em] wechselseitig[en] Zuschreibungs- und Einschätzungsprozess, begleitet und vorgeformt durch basale Gefühle der $\mathrm{Zu}$ - oder Abneigung" (Boothe und Grimmer 2005: 46). Zu diesen basalen Gefühlen gehört auch in entscheidender Weise eine narrative Vertrauensbildung: Der Patient erhält narrativen Glaubenskredit, der Therapeut kann die Erzählungen nutzen, um sich ein erstes Bild von den Darstellungsanliegen und der psychischen Konfliktdynamik seines Patienten zu machen.

Und zum Abschluss:

Die Kunst des Erzäblens besteht darin, eine Katze mit maximalem Effekt aus dem Sack zu lassen. (Hellmuth Karasek) 


\section{Literatur}

Bergmann, Jörg (1987) Klatsch: Zur Sozialform der diskreten Indiskretion. Berlin/New York: De Gruyter.

Boothe, Brigitte (2002) „Wie ist es, glücklich zu sein? Märchen zeigen, wie man in der Welt des Wunderbaren sein Glück macht“. Wie kommt man ans Ziel seiner Wünsche? Modelle des Glücks in Märchentexten. Hg. Brigitte Boothe. Gießen: Psychosozial-Verlag. 127-152.

- (2009) „Die Geburt der Psyche im elterlichen Erzählen“. Familiendynamik 34.1: $30-43$.

- (2011) Das Narrativ: Biografisches Erzäblen im psychotherapeutischen Prozess. Stuttgart: Schattauer.

- (2012) „Trieb, Wunsch und Beziehung. Psychoanalyse als Wissenschaft vom Leben“. Das Leben: Historisch-systematische Studien zur Geschichte eines Begriffs. Hgg. S. Schaede, G. Hartung und T. Kleffmann. Band 2. Siebeck: Mohr. 145183.

- (2015) „Narrative Persuasion and Narrative Irritation in Psychotherapy. Biographical Narratives, Deferred Dramaturgy and Narrative affirmation". Narrated Communities - Narrated Realities. Hgg. H. Blume, C. Leitgeb und M. Rössner. Amsterdam: Rodopi. Im Erscheinen.

Boothe, Brigitte, und Bernhard Grimmer (2005) „Die therapeutische Beziehung aus psychoanalytischer Sicht“. Die therapeutische Beziehung. Hg. Wulf Rössler. Berlin: Springer. 37-58.

Boothe, Brigitte, Simone Mosch und Georg Schönbächler (2012) „,...bewege ich doch die Unterwelt': Zur Aktualität der Psychoanalyse des Wunsches“. Psychoanalyse in Forschung und Praxis. Themenheft Psychoanalyse 16. Hgg. Brigitte Boothe, Lina Arboleda, Nicole Kapfhamer und Vera Luif. Heft 30.3/4: 356-67.

Botella, César (2005) „Die Ebenen von Erinnerung und Wahrheit: deren Deutung. Vortrag vor der Pariser Gesellschaft für Psychoanalyse“. Vortragsmanuskript.

Clandinin, D. Jean, und F. Michael Connelly (2000) Narrative Inquiry: Experience and Story in Qualitative Research. San Francisco: Jossey-Bass.

Deppermann, Arnulf (2007) Grammatik und Semantik aus gesprächsanalytischer Sicht. Linguistik - Impulse und Tendenzen 14. Berlin: De Gruyter.

Flitner, Elisabeth, und Philippe Merle (1989) „,Solange kein Fall bis zum Ende durchschaut ist...': die Psychoanalyse im Konflikt mit Freuds Verführungstheorie“. Forum der Psychoanalyse 5: 249-62.

Fludernik, Monika (2005) „Unreliability vs. Discordance. Kritische Betrachtungen zum literaturwissenschaftlichen Konzept der erzählerischen Unzuverlässigkeit“. Was stimmt denn jetzt? Unzuverlässiges Erzäblen in Literatur und Film. Hgg. Fabienne Liptay und Yvonne Wolf. München: Text \& Kritik. 39-59. 
- (2009) An Introduction to Narratology. Übers. Patricia Häusler-Greenfield und Monika Fludernik. London: Routledge.

- (2010) Towards a ,Natural' Narratology [1996]. London/New York: Routledge.

Frei, Michael, Bernhard Grimmer, Konrad Michel, Ladislav Valach und Brigitte Boothe (2012) „Gelingende und misslingende Beziehungsaufnahmen mit Patientinnen nach Suizidversuch“. Forum Qualitative Sozialforschung FQS 13.1: Artikel 5.

Freud, Sigmund (1897) Brief an Wilhelm Fliess vom 21.9.1897.

- (1952): „Uber Deckerinnerung“ [1899]. Gesammelte Werke. Bd. 1. London: Lingam Press. 531-54. http://www.freud-museum.at/freud/chronolg/1897-d.htm

- (1966) Die Traumdeutung [1900]. Frankfurt: Fischer.

- (1973) „Psychoanalytische Bemerkungen über einen autobiographisch beschriebenen Fall von Paranoia (Dementia paranoides). Darin: Über den paranoischen Mechanismus" [1911]. Studienausgabe Band VII. Frankfurt a. M.: Fischer Verlag. 183-203.

Grimmer, Bernhard (2014) Psychodynamische Gesprächskompetenzen für den Erstkontakt. Stuttgart: Kohlhammer.

Günthner, Susanne (2000) Vorwurfsaktivitäten in der Alltagsinteraktion: Grammatische, prosodische, rhetorisch-stilistische und interaktive Verfahren bei der Konstitution kommunikativer Muster und Gattungen. Tübingen: Niemeyer.

Grünbaum, Adolf (1988) Die Grundlagen der Psychoanalyse: Eine philosophische Kritik. Stuttgart: Reclam.

Hayner, Priscilla B. (2002) Unspeakable Truths: Facing the Challenge of Truth Commissions. New York: Routledge.

Hermann, Marie-Luise, Gila Rohrer, Nicole Dürler und Brigitte Boothe (2012). „Beziehungs- und Kommunikationskonzept der Kreditierung“. Psychotherapeut 58.1: 56-62.

Holt, Robert (1989) „The Present Status of Freud's Theory of the Primary Process." Freud Reappraised: A Fresh Look at Psychoanalytic Theory. Hg. Robert Holt. New York: Guilford. 280-301.

Jolles, André (1974) Einfache Formen: Legende, Sage, Mythos, Rätsel, Spruch, Kasus, Memorabile, Märchen, Witz [1930]. Tübingen: Niemeyer.

Keller, Gottfried (1879/80) „Kinderverbrechen“. Der grüne Heinrich. 2. Fassung. Stuttgart: Göschen.

Koukkou, Martha, und Dietrich Lehmann (1998) „Ein systemtheoretisch orientiertes Modell der Funktionen des menschlichen Gehirns, und die Ontogenese des Verhaltens: eine Synthese von Theorien und Daten". Erinnerung von Wirklichkeiten: Psychoanalyse und Neurowissenschaften im Dialog. Band 1: Bestandsaufnabme. Hgg. Martha Koukkou, Mrianne Leuzinger-Bohleber und Wolfgang Mertens. Stuttgart: Klett-Cotta. 287-415.

Laney, Cara, und Elizabeth F. Loftus (2005) „Traumatic Memories Are Not Necessarily Accurate Memories“. Canadian Journal of Psychiatry 50: 823-28. 
Leichsenring, Falk (2014) „Primär- und Sekundärprozess“. Handbuch psychoanalytischer Grundbegriffe. 4. erweiterte und bearbeitete Auflage. Hg. Wolfgang Mertens. Stuttgart: Kohlhammer. 742-45.

Lejeune, Philippe (1989) „Der autobiographische Pakt“. Die Autobiographie. Zu Form und Geschichte einer literarischen Gattung. Hg. Günter Niggl. Darmstadt: Wissenschaftliche Buchgesellschaft. 214-57.

Mathys, Hans-Peter, und Valérie Boucsein (2011) „Anliegen von Ratsuchenden in Abklärungsgesprächen“. Workshop an der Tagung Psychoanalyse in Forschung, Praxis und Weiterbildung. Universität Zürich, 2. September 2011.

Müller-Pozzi, Heinz (2002) Psychoanalytisches Denken - Eine Einführung. Bern: Huber.

Nawratil, Ute (2006) Glaubwürdigkeit in der sozialen Kommunikation. 2. Auflage. München. Digitale Ausgabe. <http://epub.ub.uni-muenchen.de/archive/0000 0941>

Neckel, Sighard (1986) „Das Stellhölzchen der Macht. Zur Soziologie des politischen Skandals“. Leviathan 14: 581-605.

Nünning, Vera (2010) Kulturwissenschaftliche Gedächtnisforschung und Narrationen. Vortrag am 28. April im Rahmen der 60. Lindauer Psychotherapiewochen 2010 (www.lptw.de).

- (2011) „Narrativität als interdisziplinäre Schlüsselkategorie“. Auszug aus dem Jahresbericht Marsilius-Kolleg. Universität Heidelberg. 87-104.

Nünning, Ansgar (1999) „Unreliable, Compared to What? Towards a Cognitive Theory of Unreliable Narration. Prolegomena and Hypotheses“. Grenzüberschreitungen: Narratologie im Kontext. Transcending Boundaries: Narratology in Context. Hgg. Walter Grünzweig und Andreas Solbach. Tübingen: Narr. 53-73.

Rutishauser, Armin (2007) Irritationen in psychoanalytischen Erstgesprächen - eine gesprächs-analytische Studie. Unveröffentlichte Lizentiatsarbeit. Universität Zürich.

Sarbin, Theodore (1986) Hg. Narrative Psychology: The Storied Nature of Human Conduct. London: Praeger.

Schafer, Roy (1995) Erzäbltes Leben: Narration und Dialog in der Psychoanalyse. Stuttgart: Klett-Cotta.

Spence, Donald P. (1984) Narrative Truth and Historical Truth: Meaning and Interpretation in Psychoanalysis. New York: Norton.

Streeck, Ulrich (2004) Auf den ersten Blick: Psychotherapeutische Beziehungen unter dem Mikroskop. Stuttgart: Klett-Cotta.

Wittgenstein, Ludwig (1989) Vortrag über Ethik und andere kleine Schriften. Frankfurt: Suhrkamp. (Bemerkung aus dem Jahr 1930).

Zerweck, Bernd (2004) „Unzuverlässigkeit, erzählerische“. Metzler Lexikon Literatur-und Kulturtheorie. Hg. Ansgar Nünning. Stuttgart/Weimar: Metzler. 681-82. 